\title{
Compound Odontoma in Maxillary Anterior Region: A Case Report
}

\author{
Ramya Rai, Rajashekar Reddy V*, Prashant \\ Babaji and Shashibushan KK \\ Department of Pedodontics and Preventive Dentistry, \\ Sharavathi Dental College and Hospital, India \\ *Corresponding author: Rajashekar Reddy V, \\ Department of Pedodontics and Preventive Dentistry, \\ Sharavathi Dental College and Hospital, Shimoga, \\ Karnataka, India
}

Received: February 01, 2018; Accepted: March 21 2018; Published: April 13, 2018

\begin{abstract}
Odontomas are most common hamartomatous developmental malformations. They can be differentiated into compound and complex type based on the level of organization of the tissues. Majority of odontomas are asymptomatic however these lesions can cause delayed eruption, impaction, or even retention of deciduous teeth. This case report presents a compound odontoma in 13 year old boy along with its related clinical and radiological manifestations and the surgical management.
\end{abstract}

Keywords: Odontoma; Impacted teeth; Maxilla

\section{Introduction}

Odontomas are mixed odontogenic tumors in which both the epithelial and mesenchymal components have undergone functional differentiation to the point that both enamel and dentin are formed. The enamel and dentin are typically arranged in an abnormal pattern because the organization of the odontogenic cells fails to reach a normal state of morphodiffrentiation [1]. The most common of the odontogenic tumors, odontomas are thought about as hamartomatous rather than neoplastic in nature [2].

WHO has classified odontomas into two varieties depending on their degree of morphodifferentiation. Compound odontoma is a lesion within which all the dental tissues are depicted in an orderly fashion so that there is minimum of superficial anatomic alikeness to teeth. In a complex odontoma, on the other hand, although all the dental tissues are represented, they are formed in such a rudimentary fashion that there is little or no morphologic similarity to traditional tooth formation [3].

Odontoma may be discovered at any age in any location of the dental arch with no gender predilection. Compound odontomas have a propensity for prevalence in the canine and incisor region, being found more often in the maxilla than in the mandible, whereas complex odontomas show a predilection for occurrence in the posterior jaws [4]. Compound odontomas have been reported as having a mean age of occurrence of 14.8 years compared with 20.3 years of age for complex odontomas, possibly because the odontogenict issue in the anterior jaws where the compound odontoma predominantly occurs has finished its differentiation ahead than tissues in the posterior part of the jaw [5].

They believe that the compound odontoma represents a malformation with a high degree of histomorphologic differentiation similar to the process producing supernumerary teeth, "multiple schizodontia," or locally conditioned hyper activity of the dental lamina [1].

The etiology of the odontoma is unknown, however trauma in primary dentition, Malassez paradental remnants, inflammatory processes, odontoblastic hyperactivity and hereditary anomalie [6]. It

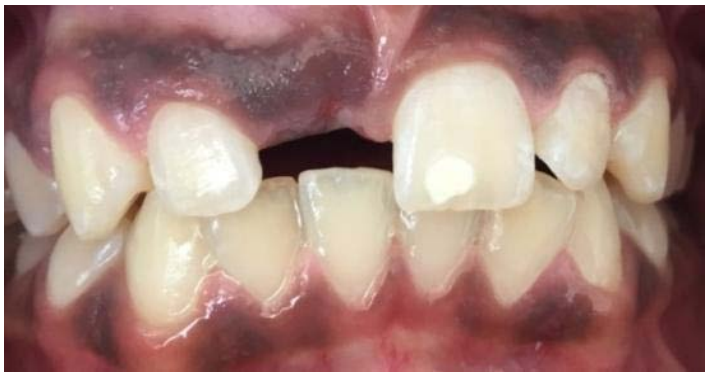

Figure 1: Preoperative photograph showing unerupted 11.

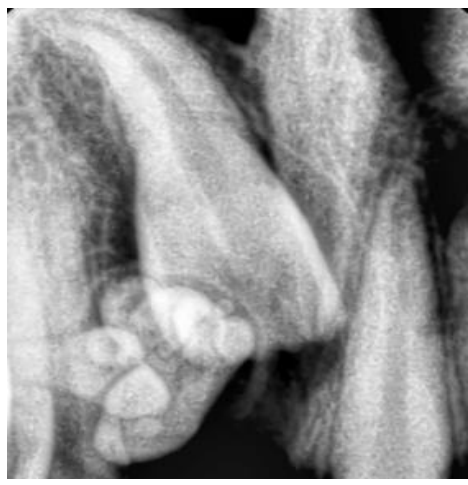

Figure 2: Radiographic evaluation using RVG.

has been suggested by Hitchin that odontomas are either inherited or are due to a mutant gene or interferences, presumably postnatal, with the genetic control of tooth development [7].

Odontomas are most commonly found on routine radiographic examination, presenting as an irregular radiopaque mass or as small, tooth like structures with the fore most frequent presenting symptom being lack of eruption of a permanent tooth or bony expansion or swelling [1].

The recommended treatment for an odontoma is conservative surgical excision, with care taken to remove the surrounding soft tissue. No propensity for recurrence has been noted [8]. 


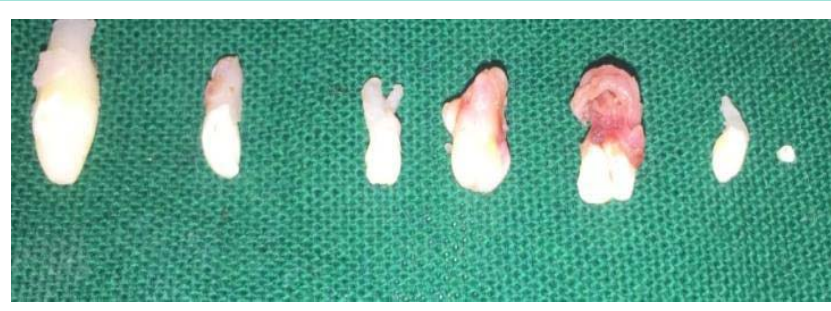

Figure 3: Seven mineralized structures removed from the lesion.

\section{Case Presentation}

A 13 year old, male patient reported with the chief complaint of unerupted upper front tooth. His medical history was not significant. Extra oral examination revealed no facial asymmetry. Intra oral examination revealed unerupted maxillary right central incisor (Figure 1). Initially a provisional diagnosis of missing 11 was done.

Intra- oral periapical radiograph revealed multiple radiopaque tooth-like structures above the crown of the unerupted permanent central incisor (Figure 2). This led to the provisional diagnosis of compound odontoma. Routine blood investigation was carried out and the patient was posted for surgery. Surgical excision of odontoma was planned under local anesthesia. Perioral structures were prepared with betadine, and a triangular mucoperiosteal flap was reflected in the regions of $11,21,22$ to expose the bone. A window was made in the bone using a straight slow speed hand piece bearing a round tungsten carbide bur under normal saline irrigation. Denticles around seven in number were removed (Figure 3 ) along with the follicle and bony sharp margins were trimmed and thorough irrigation of the enucleated site was done following the flap was repositioned and sutured (Figure 4). Post-operative follow up was uneventful (Figure $5)$.

\section{Discussion}

The term "odontoma" was coined by Pierre Paul Broca in 1867. He outlined the term odontoma as "tumors formed by the overgrowth of transitory or complete dental tissues" [6].

Odontomas are classified as a benign, mixed, calcified odontogenic tumor and are thought of in a separate category of developmental malformation rather than true neoplasm [9]. Odontomas are asymptomatic generally and gain clinical attention by inflicting delayed eruption of permanent teeth. They interfere with tooth eruption and in some cases seldom transform to cyst formation [10].

Odontomas show variation with regard to the age of occurrence. It has been suggested that although odontomas can occur at any age typically it is detected by the age of 20 yrs [11]. In the present case odontoma was diagnosed in a 13 year old male patient on a routine radiographic examination following patient's chief complaint of missing upper right front tooth. An intra-oral radiograph was taken to rule out missing permanent central incisor and revealed several tooth like structures above the crown of the unerupted permanent central incisor causing delay in the eruption of the tooth.

Reported studies show that compound odontomas often occur more frequently in the anterior maxilla in the incisor and canine region on the right side of the jaw which is confirmed in the present

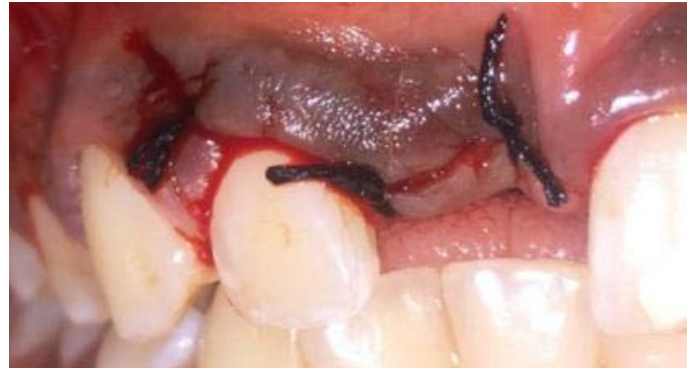

Figure 4: Suture placed.

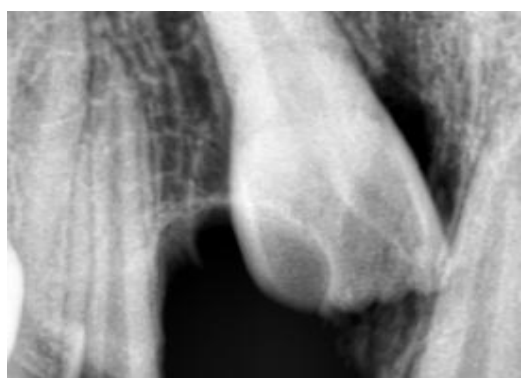

Figure 5: Postoperative radiograph

case [12].

The case described in the study was diagnosed as odontoma on the premise of clinical signs and radiographic findings. Differential diagnosis must be established with ameloblastic fibroma, ameloblastic fibroodontoma and odonto ameloblastoma. Syndromes that can be associated with odontomas are Gardner syndrome, familial colonic adenomatosis, Hermann syndrome and basal cell nevus syndrome [13].

Surgical enucleation by means of the removal of the connective tissue capsule that surrounds it is the accepted treatment of choice in order to permit the eruption of the permanent tooth. In the present case surgical exposure followed by enucleation was done number of denticles removed during enucleation of odontoma was seven in number however there are cases reported wherein 232 denticles have been removed [11], after which tooth was left in place, because it is usually recommended that in the case of an impacted tooth related to odontoma it is always better to wait for three months for the eruption of the impacted tooth. In case impacted tooth fails to erupt after three months, it is recommended for surgical exposure followed by orthodontic traction, similar method was followed in the present case [14]. A follow up was recommended to plan for the management of unerupted 11 .

\section{Conclusion}

Eruption and shedding pattern of teeth in growing children should be closely monitored by patients and delay in the eruption should be noted and consulted at the earliest. Routine investigation and screening required to find out the cause for delay. Most common cause for delayed eruption of permanent anterior teeth being fibrous growth of gingiva. One of the rare cause is odontoma its immediate surgical intervention along with orthodontic intervention with regular follow up is necessary. 


\section{References}

1. McDonald JS. Tumors of the Oral Soft Tissues and Cysts and Tumors of the Bone. In: Dean JA, Avery DR, McDonald RE. Dentistry for the Child and Adolescent, 9th ed. Mosby: Elsevier Publishers; 2010;126-149.

2. Nelson BL, Thompson LD. Compound odontoma. Head and neck pathol. 2010; 4: 290-291.

3. Siddappa A, Sunil SM, Kumar M, Selvamani M. "Compound composite odontoma associated with un-erupted permanent canine": A case report. Journal of Indian Academy of Dental specialist Researchers. 2014; 1: 74-76.

4. Paolo B, Emanuele Z, Fabio R, Cesare G. Complex and compound odontomas. J Craniofac Surg. 2012; 23: 685-688.

5. Slootweg PJ. An analysis of the interrelationship of the mixed odontogenic tumors-ameloblastic fibroma, ameloblastic fibro-odontoma, and the odontomas. Oral Surgery, Oral Medicine, Oral Pathology. 1981; 51: 266-276.

6. Barba LT, Campos DM, Rascón MM, Barrera VA, Rascón AN. Descriptive aspects of odontoma: literature review. Revista Odontológica Mexicana. 2016; 20: 265-269.

7. Das UM, Viswanath D, Azher U. A compound composite odontoma associated with unerupted permanent incisor: a case report. Int J Clin Pediatri Dent. 2009; 2: 50-55.
8. Cawson RA, Odell EW. Odontogenic tumours and tumour like lesions of the jaws. In: Essentials of Oral Pathology and Oral Medicine. 6th ed. Edinburgh: Churchill Livingstone; 1998: 117-131.

9. Kamakura S, Matsui K, Katou F, Shirai N, Kochi S, Motegi K. Surgical and orthodontic management of compound odontoma without removal of the impacted permanent tooth. Oral Surg, Oral Med, Oral Pathol, Oral Radiol, Endodo. 2002; 94: 540-542.

10. Boffano P, Zavattero E, Roccia F, Gallesio C. Complex and compound odontomas. J Craniofac Surg. 2012; 23: 685-688.

11. Uma E. Compound odontoma in anterior mandible-a case report. Malays J Med Sci. 2017; 24: 92-95.

12. Salgado H, Mesquita P. Compound odontoma- case report. Rev Port Estomatol Med Dent Cir Maxilofac. 2013; 54: 161-165.

13. Hidalgo-Sánchez O, Leco-Berrocal MI, Martínez-González JM. Meta analysis of the epidemiology and clinical manifestations of odontomas. Med Oral Pato Oral Cir Bucal. 2008; 13: 730-734.

14. Praetorius F, Piattelli A. Odontogenic tumours. In: Barnes L, Evenson JW, Reichart PA, Sindransky D, editors. WHO classification of tumours. Pathology \& genetics: Head and Neck tumours. Lyon, France: IARC Press; 2005. P. 310-311.
Austin J Dent - Volume 5 Issue 4 - 2018

ISSN : 2381-9189 | www.austinpublishing group.com

Rajashekar Reddy et al. (C) All rights are reserved
Citation: Rai R, Rajashekar Reddy V, Babaji P and Shashibushan KK. Compound Odontoma in Maxillary Anterior Region: A Case Report. Austin J Dent. 2018; 5(4): 1113. 